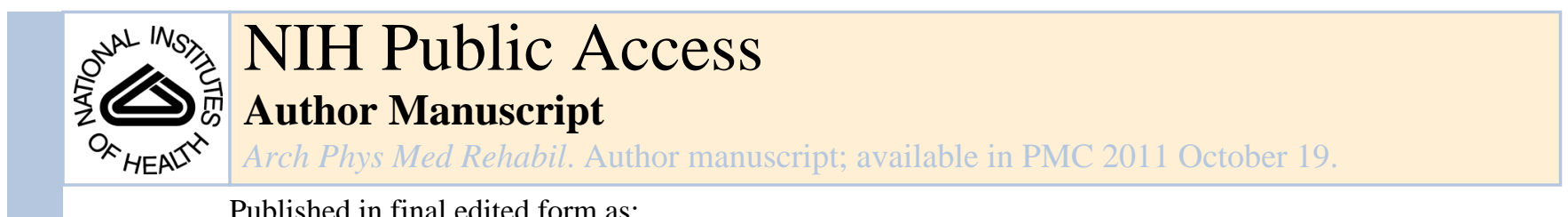

Published in final edited form as:

Arch Phys Med Rehabil. 2009 April ; 90(4): 628-633. doi:10.1016/j.apmr.2008.10.014.

\title{
Psychological factors and risk of mortality after spinal cord injury
}

\author{
James S. Krause, PhD ${ }^{1}$, Rickey Carter, PhD $^{2}$, Yusheng Zhai, MSPH $^{2}$, and Karla Reed, MA $^{1}$ \\ ${ }^{1}$ College of Health Professions, Medical University of South Carolina, Charleston, SC \\ ${ }^{2}$ Department of Biostatistics, Bioinformatics, and Epidemiology, Medical University of South \\ Carolina, Charleston, SC
}

\section{Abstract}

Objective-Our objective was to identify the association of 2 distinct psychological constructs, personality and purpose in life (PIL), with risk of early mortality among persons with spinal cord injury (SCI).

Design-Prospective cohort study with health data collected in late 1997 and early 1998 and mortality status ascertained in December 2005.

Setting-A large rehabilitation hospital in the Southeastern United States.

Participants-Adults $(\mathrm{N}=1386)$ with traumatic SCI, at least 1 year post-injury.

Interventions-Not applicable.

Main Outcome Measures-We first evaluated the significance of a single psychological predictor (a total of 6 scales) while controlling for biographic and injury predictors using Cox proportional hazards modeling and subsequently built a comprehensive model based on an optimal group of psychological variables.

Results-There were a total of $224(16.2 \%)$ observed deaths in the full sample. The total number of deaths was reduced to 164 in the final statistical model (out of 1128 participants) due to missing data. All 6 psychological factors were statistically significant in the model that was adjusted for biographic and injury factors, whereas only 3 psychological factors were retained in the final comprehensive model, including 2 personality scales (Sensation Seeking, Neuroticism-Anxiety) and the PIL scale. The final comprehensive model only modestly improved the overall prediction of survival compared to the model with only biographic and injury variables, as the pseudo- $\mathrm{R}^{2}$ increased from 0.121 to 0.129 and the concordance increased from 0.730 to 0.747 .

Conclusion-The results affirm the importance of psychological factors in relation to survival after SCI.

\section{Keywords}

Efficacy; Mortality; Personality; Rehabilitation; Risk; Spinal cord injuries

\footnotetext{
(C) 2011 The American Congress of Rehabilitation Medicine. Published by Elsevier Inc. All rights reserved

Correspondence to James S. Krause, Ph.D. Department of Rehabilitation Science College of Health Professions Medical University of South Carolina 77 President St, Suite 117 PO Box 250700 Charleston, SC 29425 Phone: 843-792-1337 Fax: 843-792-5649 krause@musc.edu Reprints are not available from this author..

Publisher's Disclaimer: This is a PDF file of an unedited manuscript that has been accepted for publication. As a service to our customers we are providing this early version of the manuscript. The manuscript will undergo copyediting, typesetting, and review of the resulting proof before it is published in its final citable form. Please note that during the production process errors may be discovered which could affect the content, and all legal disclaimers that apply to the journal pertain.
} 
One of the most disturbing long-term consequences of SCI is the elevated risk of early mortality. ${ }^{1-3}$ Unfortunately, although the mortality rate has continued to decline during the first year after SCI onset, mortality rates thereafter appear to have reached a plateau, raising the question as to the extent to which advances in medicine and rehabilitation will lead to diminished mortality in the future.

Research on causes of death after SCI provides hints as to the types of variables that may be related to early mortality. DeVivo and $\mathrm{Stover}^{4}$ calculated cause-specific SMRs to compare numbers of deaths from each cause among persons with SCI to numbers of deaths expected in the general population from those same causes. The highest SMRs suggested heightened risk of septicemia (64.2 times the general population), disease of the pulmonary circulation (47.1), pneumonia and influenza (35.6), symptoms and ill-defined conditions (13.8), and diseases of the urinary system (10.9). The causes of death that were least likely associated with SCI were homicide, legal intervention, and cancer. Because many of these causes of death are at least partially attributable to preventable factors (e.g., treatable infections, immunizations, exercise, and nutrition), this study provides indirect evidence of the importance of prevention and control of the major psychological and behavioral risk factors associated with premature mortality. In fact, prospective research on mortality has suggested that poor adaptation may be predictive of mortality as many as 15 years later. ${ }^{5}$

Krause $^{6}$ developed a model to guide research identifying empirical links between different classes of variables to mortality. The multi-stage model includes 4 levels of predictive factors for mortality, including: (1) biographic and injury factors, (2) psychological factors/ environmental factors, (3) behavioral factors, and (4) health and secondary conditions. According to the model, health factors would be the strongest predictors of mortality, followed by behavioral factors, and psychological and environmental factors. Biographic and injury factors are those factors least amenable to intervention yet the ones that have been most widely studied. At the time of the model development, prediction of mortality was virtually restricted to relationships between biographic and injury characteristics and early mortality.

In recent years, investigators have considered a wider array of factors in relation to mortality. One of the first studies to investigate a behaviorally relevant factor identified the relationship between the violent etiology of injury and early mortality utilizing data from the Model SCI Systems in the United States. ${ }^{2}$ Although a violent etiology is a behavioral factor, because it is historical in nature, it served more as a predictor for mortality than a potential target for intervention. A second study ${ }^{7}$ also used Model SCI Systems data to evaluate the general empirical risk model and found that at least one factor from each component of the model was predictive of mortality. Accounting for these variables led to substantial elevations in life expectancy under favorable circumstances (i.e., for individuals who had favorable characteristics such as fewer health problems, better insurance, and higher income). A more recent follow-up to this study, ${ }^{8}$ summarized in a brief report, suggested that life expectancy estimates might have been inflated due to instability of a single variable (workers compensation). However, because the focus of this follow-up was restricted to economic factors, there was insufficient detail provided to assess utility of the empirical risk model or to identify the significance of other types of non-biographic and injury factors in relation to mortality.

Garshick and associates ${ }^{9}$ conducted a prospective study of respiratory function among 361 men with SCI between 1994 and 2000. They identified 4 health risk factors for mortality, three of which were health status factors (diabetes, heart disease, reduced pulmonary function). They also found smoking, a behavioral factor, is associated with mortality. In 
terms of underlying and contributing cause of death, the 2 most prominent factors were diseases of the circulatory system (40\%) and diseases of the respiratory system (24\%).

A retrospective study of hospital records of all cases admitted to a Norwegian hospital between 1961 and $2002^{10}$ provided evidence for three types of risk factors for mortality: health (cardiovascular disease), behaviors (substance abuse or alcohol abuse), and psychological functioning (psychiatric disorders). The investigators emphasized the role of prevention in promoting longevity, as the 3 factors identified provide a basis for identification of those at high risk and targets for interventions (i.e., reducing cardiovascular risk factors, treating substance abuse and psychiatric disorders).

Another prospective study focused specifically on health factors and secondary conditions as predictors of mortality. ${ }^{11}$ After first controlling for biographic and injury factors (age, race, gender, injury severity), they found 5 health factors predictive of mortality in the final model that accounted for collinearity between factors. These included 1 treatment factor (hospitalizations) and 4 types of secondary conditions: (1) surgeries for pressure ulcers, (2) elevated depressive symptoms, (3) fractures/amputations, and (4) symptoms of infections. They also used 2 indices to summarize the strength of the relationship between the predictors and mortality (the pseudo $\mathrm{R}^{2}$; the $\mathrm{C}$-statistic to measure concordance). Neurologic level and ambulatory status (a proxy factor for ASIA grade) resulted in a pseudo $\mathrm{R}^{2}$ of only 0.016 and a C-statistic from .578 (.50 is chance level). Adding chronologic age, gender, and race during the next step substantially increased both the pseudo $\mathrm{R}^{2}(0.121)$ and the $\mathrm{C}$ statistic (0.730).* Adding the health and secondary conditions variables further increased the pseudo $\mathrm{R}^{2}$ to 0.178 , but only increased the C-statistic to .776 .

A second analysis of this prospective cohort data was used to evaluate the importance of behavioral factors in relation to mortality. ${ }^{12}$ The comprehensive model again was found to be superior to the basic model that included biographic and injury factors alone, as the pseudo- $\mathrm{R}^{2}$ increased from .121 in the basic model to .164 for the comprehensive model and the concordance from .152 to .191. Four behavioral predictors were significantly related to mortality in the comprehensive model including: (1) smoking, (2) binge drinking, (3) prescription medication use for pain, spasticity, sleep, or depression, and (4) time spent out of bed (a protective factor). Taken together, the findings from these studies suggest that the general empirical risk model is appropriate for guiding research of risk and protective factors for mortality but with some significant gaps in empirical research. The importance of health factors and health behaviors is most strongly established. There is some evidence for the importance of economic factors. However, with minimal exception, there is limited data on psychological factors, and that data are restricted to psychological and psychiatric disorders (e.g., depressive symptoms) which in reality are secondary health conditions. Research is needed on a broader range of psychological constructs in relation to mortality.

\section{Purpose}

Our purpose was to conduct a prospective cohort study to identify the association of 2 distinct psychological constructs with risk of mortality after SCI. These factors reflected stable traits (personality) and PIL. Biographic and injury characteristics, traditionally evaluated in mortality studies of SCI, were included in the design as primarily statistical controls. We chose the ZKPQ because it included Sensation Seeking and Neuroticism scales that may be associated with adverse outcomes after SCI, including subsequent injury. ${ }^{13} \mathrm{We}$

\footnotetext{
Race and gender were also added at the second stage of the equation, but neither is statistically significant. Therefore, they contributed minimally to any change in either parameter.
} 
anticipated that these scales would be associated with greater risk of mortality. We chose PIL because it has been associated with important SCI outcomes, including adjustment. ${ }^{14}$

\section{Hypotheses}

1

2
When statistically controlling for biographic and injury characteristics, personality and PIL will be associated with hazard of mortality.

When building an optimal risk model for mortality, inclusion of psychological factors will enhance our prediction of hazard for mortality above and beyond that of biographic and injury factors alone.

\section{METHODS}

Participants

Participants were identified from a large specialty hospital in the Southeastern United States. This hospital is one of 14 institutions currently designated as a model of care of persons with SCI by the United States Department of Education. Three hospital resources were used to identify participants including: (1) individuals in the Model SCI Systems catchment area (those routinely followed at regular intervals for whom data are contributed to a national databank), (2) Model Systems registry (rehabilitation admissions outside the catchment area), and (3) the outpatient directory (those who first come to the facility for services other than inpatient rehabilitation). To be included in the prospective cohort, participants were adults with traumatic SCI that occurred at least one year prior to the study and resulted in some residual impairment (non-neuro deficits were excluded). A total of 1929 patients were identified and 1386 participated (72\% response rate).

\section{Prospective Data Collection Procedures}

Letters introducing the study and alerting prospective participants of the forthcoming questionnaire were sent 4 to 5 weeks before the actual materials were sent. To maximize participation, 2 subsequent mailings were initiated for all non-respondents along with follow-up phone calls. Additional materials were sent out, if requested by the participant. Participants were offered a $\$ 20$ stipend and were made eligible for drawings totaling $\$ 1,500$. Data collection began July 1997 and ended April 1998, although the majority of the data had been collected by the end of 1997. All study procedures were approved by the Institutional Review Board of record prior to the study's initiation. Mortality status was determined as of December 31, 2005, as participants were either classified as deceased or presumed alive. We used the NDI of the National Center for Health Statistics ${ }^{15}$ as the primary source for determining mortality status through the end of the calendar year 2004, with the SSDI of the Social Security Administration ${ }^{16}$ used to supplement the NDI by identifying deaths occurring in 2005.*

\section{Measures}

Standardized instruments of psychological constructs including personality and PIL were used. The $\mathrm{ZKPQ}^{13}$ is a 99 -item measure of personality, which generates information on five scales (called the alternate five). These scales include: (a) Impulsive Sensation Seeking, (b) Neuroticism-Anxiety, (c) Aggression-Hostility, (d) Sociability, and (e) Activity. Impulsive Sensation Seeking was designed to measure a lack of planning and the tendency to act impulsively and served as a proxy for reckless and dangerous behavior. Neuroticism-Anxiety

A more comprehensive description of NDI and SSDI databases may be found in 11. Krause JS, Carter RE, Pickelsimer E, Wilson D. A prospective study of health and risk of mortality after spinal cord injury. Archives of Physical Medicine \& Rehabilitation 2008;89:1482-91. 
measures tension, worry, and fearfulness. Aggression-Hostility reflects items that express rude, thoughtless, or antisocial behavior. On the other hand, Activity has items describing a need for high-energy activity and Sociability reflects the number of social contacts and friends. The latter two scales served as proxies for protective behaviors required to develop needed support networks. The ZKPQ is a highly reliable instrument, with test-retest reliability ranging from .70 to .86 . Criterion-related validity values were acceptable across all scales, including correlations of 0.70 for the Impulsive Sensation Seeking scale, 0.74 for the Neuroticism-Anxiety scales, and 0.46 for the Aggression-Hostility scale (with the EASI scales: Emotionality, Activity, Sociability, and Impulsivity). ${ }^{13}$

The PIL scale ${ }^{17}$ was developed from a humanistic perspective by measuring the degree to which an individual perceives himself/herself as finding meaning in life. It consists of 20 incomplete statements rated on a 7 point scale. Scores range from 20 to 140, with participants scoring less than 92 points showing no PIL, scores between 92 and 112 showing indecisiveness, and scores over 112 showing definite PIL. This measure was included to tap whatever the individual deems to be important in their lives including many things that are not specifically addressed within this study (e.g., spirituality, presence of children, other activities). The PIL scale has been found to be highly reliable, with Pearson correlations of . 47 between PIL scale scores and therapist's ratings. Additional research has shown Pearson correlations of .81 and Spearman-Brown correlations corrected to $.92 .{ }^{18}$

\section{Statistical Considerations}

Model Building Procedures-A 3 stage hierarchical strategy to model building was employed in order to identify the association of each psychological variable with mortality and to define an optimal set of psychological predictors of mortality. Cox proportional hazards modeling was used with the number of days between the survey and event (i.e., mortality) as the dependent variable.

During the first stage of analysis, a base model consisting of biographic and injury characteristics, including functional injury classification, gender, race (Caucasian-minority), age at time of injury, and years lived since injury to the time of survey, were specified.

The second stage of the analysis focused on adding a single, psychological variable to the base model as a means of screening potential predictors for inclusion in the final stage model. All variables significant at the alpha equals 0.10 level of significance were considered for subsequent modeling. Variables that passed through the initial screening process were then assessed for multicollinearity. ${ }^{19}$

The final stage of the analysis formulated a Cox proportional hazards model that consisted of the base model and the variables identified in stage 2 of the analysis. Backwards elimination was employed to select the optimal set of predictors. Once the final model was determined, all pair wise interaction terms of the phychologic variables were included in a new model to further assess goodness of fit. A Wald linear contrast indicated no interaction item is needed $(P>0.50)$, therefore all interaction items were removed from the model.

The proportional hazards assumption of the final model was checked using the Schoenfeld residual $\mathrm{s}^{20}$ and found to be tenable. The fit of the model was assessed using the likelihood ratio test and the $\mathrm{C}$-statistic. ${ }^{21}$ The likelihood ratio test was used to calculate Nagelkerke's pseudo- $\mathrm{R}^{2}$ (a measure of model fit that indicates the proportion of the total variability explained by the model). ${ }^{22}$ The value of the C-statistic is closely related to the area under a Receiver Operating Characteristic curve and is interpretable as the probability that the cases (i.e., deaths) have higher risks as measured by the linear component of the regression model. A value of 0.5 indicates chance prediction and the discrimination of the model is improved 
as the C-value approaches 1.0. ${ }^{23,} 24$ Previous studies of mortality and SCI have identified Cvalues of approximately .730 when considering only biographic and injury factors, with this increasing to .776 after adding health predictors and .769 after adding behaviors. ${ }^{11,12}$ Therefore, anything beyond that accounted for beyond biographic and injury factors alone may be considered an improvement in fit. All model building was conducted using the SAS System version 9.1.3. The validation of the proportional hazards assumption and the estimation of the C-statistic were performed using STATA version 10.0.

\section{RESULTS}

\section{Participant Characteristics}

A total of 1386 returned usable materials ( $72 \%$ response rate). Of these, 1312 provided complete biographic and injury data and served as the base, or reference, sample for statistical analyses. Of the 74 cases removed, the majority (76\%) of the cases were removed due to insufficient information related to the injury characteristics. Missing data in the psychological constructs resulted in a smaller sample size for the final model. The final statistical model consisted of 1128 participants, 164 (15\%) of which were events.

In the reference sample, $74 \%$ were men, and $76 \%$ were Caucasian. Of the minority participants, $87.8 \%$ were African-American. Average age at time of injury was 31.4 years (IQR: 20.6 to 38.9). At time of prospective data collection, the participants' mean age was 40.3 years (IQR: 30.1 to 48.4 ), and they had been injured a mean of 8.9 years (IQR: 3.5 to $12.3)$. The primary etiology was vehicular crashes $(51 \%)$, followed by falls/flying objects $(17 \%)$, acts of violence (13\%), sports (12\%), and other (7\%).

Fifty-four percent reported cervical injuries, and $21 \%$ reported ability to ambulate. Functional injury classification was defined according to a combination of injury level and ambulatory status that yielded 5 categories that were similar, but not equivalent, to those frequently reported in the SCI mortality literature.* Thirteen percent had upper cervical injuries (C1-C4) and were non-ambulatory; $31 \%$ had a lower cervical injury (C5-C8) and were non-ambulatory; $35 \%$ were non-ambulatory with non-cervical injuries; $11 \%$ had a cervical injury but were ambulatory; and the remaining $10 \%$ had non-cervical injuries and were ambulatory.

\section{Modeling}

Table 1 summarizes the results of statistical modeling. It includes an analysis of the raw relationship of the biographic, injury related, and psychological variables with mortality, followed by consideration of the psychological variables evaluated after controlling for the biographic and injury characteristics. At last, the final model is summarized evaluating all biographic, injury related, and retained psychological variables simultaneously.

With all biographic and injury related factors in the model, significant HRs were observed for age at injury onset (HR=1.06, CI: 1.05, 1.07), years lived since injury $(\mathrm{HR}=1.05, \mathrm{CI}$ : $1.03,1.07)$, and injury severity. According to recent research reports, we broke down the injury severity into 5 categories, with 2 ambulatory groups -- cervical and noncervical. The 2 ambulatory groups were not significant different from each other, although the 3 other groups were significantly different from the noncervical ambulatory group (the reference group). Participants with the most severe injuries (C1-C4, non-ambulatory) had the greatest hazard (HR=4.83, CI: $2.33,10.00)$. The 2 other groups with non-ambulatory injuries (C5-

Convention has been to use four groups based on the breakdown according to the three levels for ASIA grades AC, with a single group denoting ASIA-Dregardless of injury level. We have used ambulatory status in lieu of ASIA grades which are not available and have broken this down according to cervical and non-cervical injuries. 
C8; noncervical) also had significantly elevated HRs compared with those with the least severe injuries. However, 2 ratios were observed modestly reversed from what would be expected based on injury severity $(\mathrm{C} 5-\mathrm{C} 8=3.13$, non-cervical $=3.41)$, suggesting substantial similarities between these 2 groups. The 2 ambulatory groups had essentially identical hazards of mortality. Race and gender were retained in the subsequent adjusted model to account for their potential confounding effects, though none of them were significant.

All 5 personality scales and the PIL scale achieved the screening criterion for inclusion in the model building steps. The final model yielded 2 of the 5 personality indicators:

Sensation Seeking (HR=1.02, CI: 1.00, 1.04) and Neuroticism-Anxiety (HR=1.02, CI: 1.00, 1.04), and the PIL scale (HR=0.99, CI: $0.98,1.00)$. Since all 3 variables were scores, to better interpret their relationship with the hazard of mortality, we introduced the standardized HR. The mean (SD) of Sensation Seeking was 49.95 (10.05) which gave an estimated standardized HR of 1.22. This value may be interpreted as the hazard of mortality increases by $22 \%$ for every 1 SD (10.05 units) increase in Sensation Seeking when all other variables were held constant in the final model. The standardized HR of NeuroticismAnxiety was calculated as 1.21 given its mean (SD) was 49.95(9.98). This value may be interpreted as the hazard of mortality increases by $21 \%$ for every 1 SD (9.98 units) increase in Neuroticism-Anxiety when all other variables in the final model were held constant. The mean (SD) of PIL was 99.36 (21.04), thus, a standardized HR of 0.81 was obtained. This value may be interpreted as the hazard of mortality decreases by $19 \%$ for every 1 SD (21.04 units) increase in PIL when all other variables in the final model were held constant.

Table 2 demonstrates the comparison of the pseudo- $\mathrm{R}^{2}$ and the $\mathrm{C}$-statistic through the model developing process. The base model with only the injury and the biographic factors as predictors has a pseudo- $\mathrm{R}^{2}$ of only 0.121 and a concordance of only 0.730 . The maximum model created by adding all significant psychological predictors to the base model offers a slight increase on both indicators $\left(\mathrm{R}^{2}=0.137, \mathrm{C}=0.754\right)$. The final model combined injury and biographic factors with only the psychological predictors retained from the backward selection process of the full model, and had a negligible decrease in both pseudo- $R^{2}\left(R^{2}=\right.$ $0.129)$ and concordance $(C=0.747)$ compared with the full model.

\section{DISCUSSION}

The unique contribution of this study is the identification of the association of psychological characteristics with mortality after SCI using prospective cohort design and a priori selection of variables using a general risk model. Two types of psychological characteristics were investigated in relation to mortality, each reflecting different theoretical constructs. Whereas personality is deemed to be stable and associated with consistent patterns of behavior that are highly resistant to change, PIL represents the extent to which the individual has found meaning in her/his life and, in theory, may change through multiple types of activities that either introduce meaningful identities into life or address appraisals of the current life situation.

From the standpoint of prediction, personality traits may be optimal since they are, in theory, stable, whereas PIL may be better in relation to change. Although all characteristics were significantly associated with mortality, only 2 personality characteristics and PIL were included in the final model. Therefore, these are the most logical candidates for both prediction and prevention.

Given the nature of the 3 characteristics that were significantly associated with mortality, it is likely that individuals who habitually take risks will likely continue to be at high risk for early mortality. This is problematic given the fact that high-risk behaviors often lead to 
SCI. ${ }^{25,} 26$ Therefore, there is a continuity of risk behaviors leading to injury and post-injury patterns of behavior that are associated with elevated risk for mortality. The presence of Neuroticism-Anxiety is associated with further risk of mortality. Since this scale measures tension, worry, and fearfulness, it is possible that this reflects a pattern of apprehensiveness and affects the ability to adjust to SCI. The mechanisms by which personality may be related to mortality are not clear, nor are they addressed in this study.

PIL is an interesting construct, as it reflects an individual's ability to find meaning. In essence, individuals high on this scale feel their life is purposeful, they are more likely to have goals, and they find their lives interesting on a daily basis. The PIL scale is not tied to any specific area of life (e.g., employment, church, family), but rather one's overall appraisal of meaning in life, so there are multiple ways of finding meaning in life, and this is associated with greater longevity. However, we cannot determine from the current study whether finding PIL is associated with a pattern of behaviors, such as greater activities and diminished likelihood of abusing alcohol or prescription medications, which actually promote greater longevity. More sophisticated mediational models will be needed to identify these types of relationships.

A review of the pseudo- $\mathrm{R}^{2}$ and $\mathrm{C}$-statistic suggests there is a great deal that one needs to learn about psychological factors in the prediction of mortality. Neither the pseudo- $\mathrm{R}^{2}$ for the full model $\left(\mathrm{R}^{2}=0.129\right)$ nor the $\mathrm{C}$-statistic $(\mathrm{C}=0.747)$ represented substantial improvements over the base model $\left(R^{2}=0.121 ; C=0.730\right)$ and both were lower than that from previous studies that used health factors ${ }^{11}$ and health behaviors ${ }^{12}$ as predictors. The net effect of these variables would be only about 2 years of life expectancy for a younger individual.

\section{Implications}

Identification of psychological risk and protective factors in relation to mortality has 2 direct implications for intervention. First, assessment of characteristics that are associated with a greater risk of mortality will help identify those individuals who are at greatest risk of early mortality. A minimal intervention is to share that information with the individual which will in turn empower the individual to make informed choices about his/her life.

Second, psychological factors may represent targets for intervention. However, some psychological factors, particularly personality traits, by definition are resistant to change. Therefore, the focus of such interventions may better be directed to other characteristics, such as finding meaning or PIL. Because meaning can come from multiple sources (e.g., gainful employment, family, spirituality, volunteerism), there are a wide range of intervention options.

However, it must be pointed out that strength of the relationships between psychological factors in this study and mortality was modest at best, so interventions would not likely change life expectancy substantially. Given the relatively weak associations between the psychological predictors and mortality, life expectancy would vary minimally based on the characteristics within this study. This of course assumes that the strength of association is not compromised by the interval of time between assessment and determination of mortality status. Even though personality is theoretically stable, we would anticipate some changes in personality over time and more substantial changes in PIL. We also used a non-pathological measure of personality and did not measure more pertinent psychological factors, such as personality disorders, that may have had greater explanatory power given their potential association with adverse psychological states and maladaptive behavioral patterns. 


\section{Study Limitations}

The primary limitations of the study include: (1) left censoring of the data, (2) absence of data in the first year when mortality is highest, (3) utilization of only a subset of potential psychological measures, and (4) potential influence of missing data on estimating life expectancy and the strength of predictors. Of these four limitations, the first (left censoring) is probably the most significant. This occurs when the sample is drawn from some point after inception (the time of the SCI), and it results in cases never being enrolled in the study, some of which could have occurred due to mortality. We do not know whether there are systematic differences between those who lived to participate and potential participants who died prior to initiation of the data collection. The absence of data in the first year post-injury is probably not as critical, since our focus was on psychological factors which one would think would be related to more long-term outcomes. Third, we utilized only a subset of psychological measures and constructs that could have been be used to identify risk for early mortality. An inherent difficulty in prospective studies of mortality is that conceptual frameworks become more or less popular over time, and there is no opportunity to change based on recent trends. Lastly, there was a fair amount of missing data which resulted in dropping some participants from the analyses and a disproportionate percentage of deceased cases were dropped. This could potentially result in overestimating life expectancy but also could have led to lower estimates for HRs of individual predictors.

\section{Future Research}

Future research would benefit from several types of study enhancements, including multiple points of data collection prior to assessment of mortality, assessment of causes of death, and testing of a more comprehensive model that includes other types of factors in relation to early mortality, such as environmental, behavioral, and health factors. It is also time to move towards intervention based research to enhance longevity after SCI.

\section{CONCLUSIONS}

Using a prospective cohort design guided by a general empirical risk model, we identified 3 psychological variables, including 2 personality scales (Sensation Seeking and NeuroticismAnxiety) and the PIL scale that were related to mortality. Assessing these constructs in clinical settings will help to identify individuals at high risk for early mortality, as well as provide targets for prevention strategies.

\section{Acknowledgments}

Supported by a field initiated grant from the National Institute for Disability and Rehabilitation Research (grant no. H133G030117), and the Model Spinal Cord Injury Systems (grant no. H133N000005). The opinions here are those of the grantee and do not necessarily reflect those of the funding agencies.

No commercial party having a direct financial interest in the results of the research supporting this article has or will confer a benefit on the authors or on any organization with which the authors are associated.

Sponsorship: This research was supported by a field initiated grant from the National Institute for Disability and Rehabilitation Research (H133G030117) and the Model Spinal Cord Injury Systems Grant \#H133N000005.The opinions here are those of the grantee and do not necessarily reflect those of the funding agencies.

I certify that no party having a direct interest in the results of the research supporting this article has or will confer a benefit on me or on any organization with which I am associated AND, if applicable, I certify that all financial and material support for this research (eg, NIH or NHS grants) and work are clearly identified in the title page of the manuscript. 


\section{List of Abbreviations}

$\begin{array}{ll}\text { HR } & \text { hazard ratio } \\ \text { IQR } & \text { interquartile range } \\ \text { NDI } & \text { National Death Index } \\ \text { PIL } & \text { Purpose in Life } \\ \text { SSDI } & \text { Social Security Death Index } \\ \text { SCI } & \text { spinal cord injury } \\ \text { SMR } & \text { standardized mortality ratio } \\ \text { ZKPQ } & \text { Zuckerman-Kuhlman Personality Questionnaire }\end{array}$

\section{References}

1. National Spinal Cord Injury Statistical Center. Annual Statistical Report. University of Alabama; Birmingham, AL: June. 20062006

2. DeVivo MJ, Krause JS, Lammertse DP. Recent trends in mortality and causes of death among persons with spinal cord injury. Arch Phys Med Rehabil. 1999; 80:1411-9. [PubMed: 10569435]

3. Strauss DJ, DeVivo MJ, Paculdo DR, Shavelle RM. Trends in life expectancy after spinal cord injury. Arch Phys Med Rehabil. 2006; 87:1079-85. [PubMed: 16876553]

4. DeVivo, MJ.; Stover, SL. Long term survival and causes of death. In: Stover, SL.; DeLisa, JA.; Whiteneck, GG., editors. Spinal cord injury: clinical outcomes from the model systems. Aspen; Gaithersburg, MD: 1995. p. 289-316.

5. Krause JS. Survival following spinal cord injury: A fifteen-year prospective study. Rehabil Psychol. 1991; 36:89-98.

6. Krause JS. Secondary conditions and spinal cord injury: A model for prediction and prevention. Top Spinal Cord Inj Rehabil. 1996; 2:217-27.

7. Krause J, Devivo M, Jackson A. Risk factors for mortality after spinal cord injury. Arch Phys Med Rehabil. 2004; 85:1764-73. [PubMed: 15520971]

8. Strauss D, DeVivo M, Shavelle R, Brooks J, Paculdo D. Economic factors and longevity in spinal cord injury: a reappraisal. Arch Phys Med Rehabil. 2008; 89:572-4. [PubMed: 18295640]

9. Garshick E, Kelley A, Cohen SA, et al. A prospective assessment of mortality in chronic spinal cord injury. Spinal Cord. 2005; 43:408-16. [PubMed: 15711609]

10. Lidal I, Snekkevik H, Aamodt G, Hjeltnes N, Biering-Sorensen F, Stanghelle J. Mortality after spinal cord injury in Norway. Journal of Rehabilitation Medicine. 2007; 39:145-51. [PubMed: 17351697]

11. Krause JS, Carter RE, Pickelsimer E, Wilson D. A prospective study of health and risk of mortality after spinal cord injury. Arch Phys Med Rehabil. 2008; 89:1482-91. [PubMed: 18674984]

12. Krause JS, Carter RE, Pickelsimer E. Behavioral risk factors for mortality after spinal cord injury. Arch Phys Med Rehabil. in press.

13. Zuckerman M, Kuhlman DM, Joireman J, Teta P, Kraft M. A comparison of three structural models for personality, The Big Three, The Big Five and The Alternate Five. J Pers Soc Psychol. 1993; 65:757-68.

14. Thompson N, Coker J, Krause JS, Henry E. Purpose in life as a mediator of adjustment after spinal cord injury. Rehabil Psychol. 2003; 48:100-8.

15. [Accessed September 28, 2007] National Death Index. Available from: URL: http://www.cdc.gov.nchs/ndi.htm.

16. [Accessed September 28, 2007] Social Security Death Index. Available from: URL:http://www.ancestry.com.

17. Crumbaugh JC. Cross-validation of Purpose-In-Life test based on Frankl's concepts. J Individ Psychol. 1968; 24:74-81. [PubMed: 4385494] 
18. Crumbaugh, JC.; Maholick, LT. Manual of instructions for the Purpose in Life Test. Institute of Logotherapy; Saratoga, CA: 1981.

19. Hosmer, DW.; Lemeshow, S. Applied logistic regression. 2nd ed.. John Wiley and Sons; New York, New York: 2000.

20. Schoenfield, d. Chi-squared goodness-of-fit tests for the proportional hazards regression model. Biometrika. 1980; 67:145-53.

21. Harrell FE Jr, Lee KL, D.B. M. Multivariate prognostic models: Issues in developing models, evaluating assumptions and adequacy, and measuring and reducing errors. Stat Med. 1996; 15:361-87. [PubMed: 8668867]

22. Nagelkerke N. A note on general definition of the coefficient of determination. Biometrika. 1991; 78:691-2.

23. Harrell FE Jr, Califf RM, Pryor DB, Lee KL, Rosati RA. Evaluating the yield of medical tests. JAMA. 1982; 247:2543-6. [PubMed: 7069920]

24. Harrell, FE, Jr. Regression modeling strategies with applications to linear models, logistic regression, and survival analysis. Springer-Verlag New York Inc.; New York: 2001.

25. Fordyce WE. Personality Characteristics in men with spinal cord injury as related to manner of onset of disability. Arch Phys Med Rehabil. 1964; 45:321-5. [PubMed: 14180432]

26. Rohe DE, Krause JS. The five-factor model of personality: findings in males with spinal cord injury. Assessment. 1999; 6:203-14. [PubMed: 10445958] 


\section{Table 2}

Model Fit Statistics

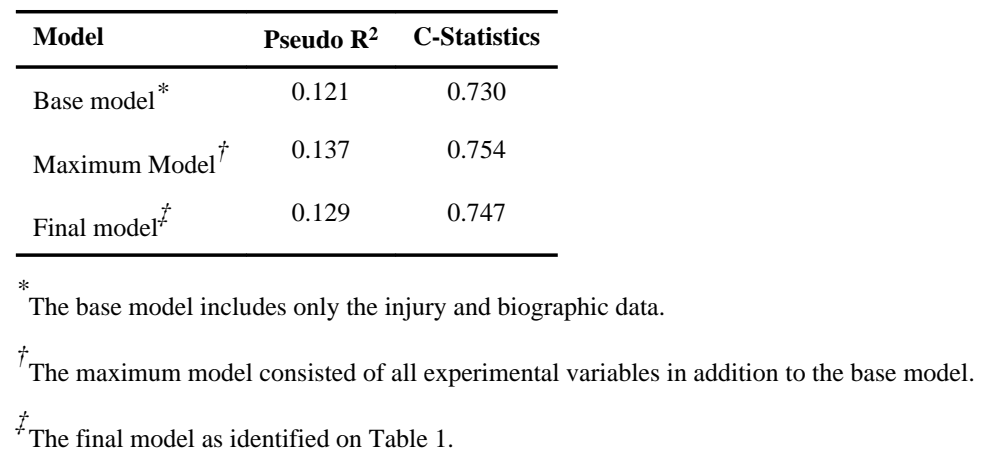

